Because Xie wanted to investigate the relationship between transcription and translation system-wide in single molecules, he also needed a way to count the mRNA molecules encoding the YFPtagged protein in each strain. Using fluorescence in situ hybridization, graduate student Gene-Wei Li could detect an individual $y f p$ mRNA labeled with a single red fluorophore in a fixed cell.

While Taniguchi collected protein expression data in live cells for all 1,018 strains, Li used the library and RNA counting to simultaneously measure mRNA and protein levels in single fixed cells for 137 of the more highly expressing strains.

"This is the first time the proteome has been characterized at single-molecule sensitivity in any organism," remarks Xie. They found that half of all the proteins are present at fewer than ten molecules per cell and $20 \%$ of proteins are expressed at under one copy per cell. Xie admits that some were leakages, but others were functioning proteins. They found that the protein expression is represented by a gamma distribution and, perhaps surprisingly, there was no correlation between the mRNA copy number and protein copy number in the same cell. This is explained by the stochastic nature of transcription and the fact that most of the proteins had a lifetime longer than the cell cycle, whereas the mRNAs had a much shorter lifetime.

These results promise to be the first of many biological insights to come. Xie is making the library available for other people to use and is looking forward to applying single-molecule systems biology to questions such as bacterial persistence.

\section{Daniel Evanko}

\section{RESEARCH PAPERS}

Taniguchi, Y. et al. Quantifying $E$. coli proteome and transcriptome with singlemolecule sensitivity in single cells. Science 329, 533-538 (2010).

Not only does the formulation of Kussell and Leibler provide a tool to study the effective strength of selection in a particular system, it also allows one to infer whether a cell or unicellular organism is using a stochastic switch to adapt to a particular environmental fluctuation. "For stochastic switches, there is a very pronounced peak in the historical fitness variance, which occurs at fluctuation periods that are longer than the generation time," says Kussell. "So you can infer the existence and the type of an internal mechanism when you essentially don't know what the organism is doing, and you don't know what genes are important." Undoubtedly, it will be of interest to begin to dissect the contribution of particular genes to such predicted stochastic switches.

In this work, Kussell and Leibler used lineage data obtained from simulations of growing Escherichia coli. However, experimental data in which growing cells, either microorganisms or mammalian cells, are imaged over time by live-cell video microscopy are increasingly becoming available. The theory developed in this work may well prove useful for studying the mechanisms by which populations of dynamic cells, stem cells or tumor cells, for instance, change in response to the environment, in contexts in which there is a selective advantage for the cells to be in one state or another. Natalie de Souza

\section{RESEARCH PAPERS}

Leibler, S. \& Kussell, E. Individual histories and selection in heterogenous populations. Proc. Natl. Acad. Sci. USA 107, 13183-13188 (2010).

\section{NEWS IN BRIEF}

\section{BIOCHEMISTRY}

\section{iCLIP at nucleotide resolution}

Most, if not all, human mRNAs are spliced into several isoforms by heterogeneous nuclear ribonucleoprotein (hnRNP) particles that associate with the nascent transcript. Koenig et al. now precisely map binding of an hnRNP by a twist on UV-light cross-linking and immunoprecipitation (CLIP). In individualnucleotide-resolution CLIP (iCLIP), each RNA reveals the exact site of protein binding. These data will yield insight into the effect of hnRNP binding on splicing.

Koenig, J. et al. Nat. Struct. Mol. Biol. 7, 909-915 (2010).

\section{PROTEOMICS}

\section{0-glycosylation stoichiometry}

Sugar-based post-translational modifications remain a challenge to characterize, owing to their low abundance and diverse chemistries. Rexach et al. now describe a straightforward approach to characterize the stoichiometry and dynamics of 0 -GICNAC glycosylation in vivo, by selectively labeling terminal 0 -GlcNAc sugars with a polyethylene glycol-based mass tag that can be easily detected by denaturing polyacrylamide electrophoresis and immunoblotting. This allowed them to follow the interplay of phosphorylation and glycosylation in mammalian cells. Rexach, J.E. et al. Nat. Chem. Biol. advance online publication (25 July 2010).

\section{STEM CELLS}

\section{Changing substrate rigidity to grow stem cells}

There is increasing interest in understanding how mechanical properties of the growth substrate influence cellular biology. Gilbert et al. now show that muscle stem cells can be cultured for one week on hydrogel surfaces with a rigidity that matches that of muscle. In contrast to rigid surfaces, the soft surface promotes stem cell self-renewal and maintains the ability of the cells to regenerate muscle when transplanted in vivo. Whether the effect persists for culture over longer periods awaits further study. Gilbert, P.M. Science advance online publication (15 July 2010).

\section{MICROSCOPY}

\section{tRNA movement through the ribosome}

Time-resolved, single-particle electron cryomicroscopy allows biological processes to be followed by taking multiple snapshots of dynamic systems, but a very large computational effort is required to sort images of heterogeneous complexes. By taking and analyzing two million images of tRNA movement through the ribosome at various time points, Fischer et al. used this technique to observe 50 distinct ribosome sub-states, gaining insights into the mechanism of the translocation step of protein synthesis. Fischer, N. et al. Nature 466, 329-333 (2010).

\section{PROTEOMICS}

\section{Characterizing protein ubiquitination}

The low abundance of ubiquitination sites make them difficult to detect. Xu et al. now describe a method to enrich these previously ubiquitinated peptides with a monoclonal antibody reagent that recognizes a unique diglycine adduct left on modified lysine residues after tryptic digestion. Using mass spectrometry, they identified 374 ubiquitination sites on 236 human proteins, adding substantially to the number of known ubiquitination sites. Xu, G. et al. Nat. Biotechnol. advance online publication (18 July 2010). 